\title{
28 Research Soure \\ Validity and reliability of the Finnish Motivation for Physical Activity (RM4-FM) questionnaire
}

\section{Mikko Uimonen}

Central Finland Centrl Hospital

Jussi P. Repo

Keski-Suomen keskussairaala

Kiira Grönroos

Jyvaskylan yliopisto Liikuntatieteellinen tiedekunta

Arja Häkkinen

Jyvaskylan yliopisto Liikuntatieteellinen tiedekunta

Simon Walker ( $\square$ simon.walker@jyu.fi )

University of Jyväskylä https://orcid.org/0000-0002-6804-0741

\section{Research article}

Keywords: Self-determination theory, Inactivity, Psychometric properties, Patient reported outcome measure

Posted Date: January 13th, 2020

DOl: https://doi.org/10.21203/rs.2.20652/v1

License: (c) (i) This work is licensed under a Creative Commons Attribution 4.0 International License.

Read Full License 


\section{Abstract}

Background There is a lack of properly validated instruments measuring motivation for physical activity in the Finnish language. The study aimed to translate the Motivation for Physical Activity (RM4-FM) instrument into Finnish and examine its psychometric properties in a sample of healthy, older Finnish adults.

Methods The RM4-FM was translated and linguistically validated adhering to published guidelines. The sample consisted of 102 65-75-year old participants, who completed the RM4-FM, the Physical Activity Acceptance Questionnaire (PAAQ) and sociodemographic characteristics questionnaires electronically. The RM4-FM was re-administered one month after the initial assessment.

Results RM4-FM translated well into Finnish. The motivation dimension scores were skewed towards high internal and low external motivation. A floor effect was confirmed in the External regulation dimension and a ceiling effect was confirmed in the Identified regulation dimension. Confirmatory factor analysis indicated inadequacy of the four-dimension model. Separate assessment of each of the four motivation dimensions with Exploratory factor analysis showed unidimensionality for all dimensions. Exploratory factor analysis provided a best-fit model of three factors (influence of other people, intention of well-being and emotional aspect of motivation). Re-administration of the instruments showed good test-retest reliability in all motivation dimensions. Female gender, higher education and higher PAAQ score were associated to higher internal motivation and lower external motivation scores.

Conclusions The Finnish version of the RM4-FM instrument provides valid and reliable scores in assessing motivation for physical activity in a sample of healthy, older Finnish adults. The score distributions suggested shortages in the scale when evaluating internally motivated subjects. The threefactor model of the RM4-FM provided more favorable structural validity compared to the original version. This work may aid in future translations into other languages and uses of the questionnaire.

\section{Background}

Physical activity has a key role promoting well-being and quality of life ${ }^{1}$. Inactivity has been shown to be a major risk factor for several chronic diseases, such as cardiovascular diseases, diabetes mellitus type 2, osteoporosis and colon cancer as well as psychological disorders ${ }^{25}$. The prevalence of such diseases in western countries is high 5,11,12,15,30,34. In addition, cardiovascular diseases are a major cause of death 30,34 . Overall, the burden of such diseases for society are substantial measured in costs and healthy years lived ${ }^{3,38}$. The evidence of the positive effects of physical activity on the risk and, on the other hand, the prognosis of the chronic diseases is indisputable ${ }^{26}$. Despite these positive effects on health, inactivity is still a major issue in public health 2,16 .

On an individual level, a key point of the decision of whether to be physically active is motivation. The Self-determination theory by Deci and Ryan ${ }^{9}$ divides motivation into controlled and autonomous 
aspects. In the field of physical activity, the autonomous aspect of motivation refers to the intention of joy, well-being and achievement of personal goals derived from physical activity, while the controlled aspect refers to the influence and the pressure of others, as well as the guilt of not exercising ${ }^{9}$. Selfdetermination theory proposes the importance of the motivational factors originating from oneself, rather than external factors, on the determination between physically active or physically inactive lifestyles. Strong intrinsic motivation has been shown to be associated with positive long-term effects of physical activity, as intrinsically motivated individuals continue being active even when the influence of external pressure has stopped ${ }^{932}$. Thus, to achieve permanent positive change in one's physical activity habits, it is crucial to enhance the intrinsic motivation aspect.

Lately, the development of interventions on strengthening the motivation for physical activity has been a subject of interest in the field of sport science and medicine $10,29,31,36$. Such interventions have been effective in enhancing internal motivation for physical activity ${ }^{10,31}$. However, it is important to identify the individuals who are at risk for physical inactivity-related diseases and have issues with their motivation to provide targeted interventions to those who benefit most. For this purpose, several questionnaires have been developed ${ }^{7,21,23}$.

The Motivation for Physical Activity (RM4-FM) questionnaire is a self-administered instrument developed to assess the internal and external aspects of physical activity motivation ${ }^{7}$. RM4-FM was developed based on Self-determination theory. RM4-FM has been utilized to examine motivation for physical activity 24 , yet it has not been properly validated for this purpose. In addition, modified versions of RM4-FM have been used in assessing motivation for dieting and quitting smoking ${ }^{17}$.

Validation and understanding of psychometric properties of measurement instruments is indispensable for correct use of these questionnaires ${ }^{19,20}$. Proper validation processes enable researchers to evaluate the usability of the instrument when assessing whether to choose the instrument when initiating a new study. Additionally, proper validation creates the frames for interpreting and assessing the meaning of the results obtained from these instruments.

Hitherto, the psychometric properties of the RM4-FM questionnaire have not been examined properly. In addition, the instrument has not been translated or validated for the Finnish population. The aim of the present study was to translate the RM4-FM instrument into Finnish and examine the psychometric properties of the translated version in a sample of healthy, older adults.

\section{Methods}

The study was conducted as part of an exercise intervention study examining the effect of resistance training frequency on strength, functional capacity and health outcomes (NCT02413112). The study contacted two-thousand 65-75-year old people living in the Jyväskylä city region by recruitment mail, who were identified based on age and selected randomly from the Population Register. Those who were willing to participate to the study $(n=454)$ completed an electronic preliminary registration questionnaire 
that was used to select individuals to an information meeting $(n=148)$. The individuals with health issues that may hamper involvement to physical activity (notable overweight with BMI 37 or above, poorly controlled cardiovascular disease or lower extremity injury that may complicate physical training and testing, a need of assistive device, use of medication that influences the neurological or endocrinal functions) or who already involved regularly in physical exercise or sport (over 180 minutes per week) or had other factors which may affect the physical performance and exercise (previous experience of strength training, previous testosterone treatment, smoking) were excluded. After all, 102 suitable individuals signed informed consent to participate and were included to the study. The baseline characteristics of the participants are shown in Table 1. The participants completed the RM4-FM and the Physical Activity Acceptance Questionnaire (PAAQ) questionnaires electronically, as well as a questionnaire on sociodemographic characteristics. The participants were asked to re-complete the RM4FM questionnaire one month after the initial assessment. 
Table 1

Sociodemographic and clinical characteristics of the participants.

\begin{tabular}{|lc|}
\hline & $\mathrm{N}=102$ \\
\hline Women, $\mathrm{n}(\%)$ & $57(56)$ \\
\hline Age, mean (SD) years & $69(3)$ \\
\hline BMl, mean (SD) kg/m² & $28(4)$ \\
\hline Physical activity, mean (SD) minutes per week & $104(61)$ \\
\hline Education, $\mathrm{n}(\%)$ & \\
\hline Basic education or general upper secondary & $65(64)$ \\
\hline University or college & $37(36)$ \\
\hline Marital status, $\mathrm{n}(\%)$ & $79(77)$ \\
\hline Married or living with the spouse & $23(23)$ \\
\hline Divorced, widow or unmarried & \\
\hline Chronic diseases, $\mathrm{n}(\%)$ & $32(31)$ \\
\hline Cardiovascular & $10(10)$ \\
\hline Respiratory & $11(11)$ \\
\hline Musculoskeletal & $7(7)$ \\
\hline Diabetes & $3(3)$ \\
\hline Gastrointestinal & $3(3)$ \\
\hline Neurological & $17(17)$ \\
\hline Other & $52(51)$ \\
\hline Using medication, $\mathrm{n}(\%)$ & 50 \\
\hline Physical Activity Acceptance Questionnaire (PAAQ) score, mean (SD) & \\
\hline
\end{tabular}

\section{Instruments}

\section{RM4-FM: Motivation for Physical Activity}

RM4-FM is a 16-item questionnaire measuring the four dimensions of motivation for physical activity ${ }^{7}$. All items use a seven-point Likert-scale. The RM4-FM is divided in four dimensions of four items: External regulation, Introjected regulation, Identified regulation, and Intrinsic motivation. The score of each dimension is calculated as a mean of the scores of that dimension's items. Higher scores indicate 
stronger influence of motivation within the specific dimension. In addition, the Relative Autonomy Index (RAI) is calculated by using a formula including all four motivation dimension scores (RAI $=2 \times$ Intrinsic motivation + Identified regulation - Introjected regulation $-2 \times$ External regulation). RAl indicates the relative impact of intrinsic and extrinsic factors in motivation for physical activity. A negative RAI value indicates that extrinsic factors have stronger influence on motivation while a positive RAI value indicates stronger influence of intrinsic factors on motivation, and thus autonomy.

\section{Physical Activity Acceptance Questionnaire (PAAQ)}

The PAAQ is a 10-item questionnaire measuring the subjective psychological and physical discomfort caused by physical activity ${ }^{4}$. All items use a seven-point Likert-scale and the maximum score is 70 . Lower scores indicate higher amount of discomfort caused by physical activity and problems with motivation to continue such activity. The PAAQ has been shown to be valid and reliable in measuring psychological factors behind motivation for physical activity ${ }^{4}$.

\section{Translation and cultural adaptation process}

The translation process and linguistic validation of the RM4-FM questionnaire adhered to the guidelines of Wild et al. (2005) ${ }^{37}$. Two native Finnish academic researchers of sport science independently forwardtranslated the RM4-FM into Finnish language. These two translated versions were then compared and reconciliated. The reconciliated version was then back-translated to English by a native English-speaking researcher that is also fluent in Finnish. After back-translation, discrepancies between forward- and backtranslated versions of RM4-FM were corrected and accordance between the original and translated versions was verified. Finally, all researchers that participated in the translation process proofread and approved the final version.

\section{Statistical analysis}

The sociodemographic and clinical data are presented as means, standard deviations (SD) and $95 \%$ confidence intervals $(\mathrm{Cl})$ or as counts with percentages. Normality of the RM4-FM and its dimensions score distributions were assessed. Floor and ceiling effects were examined from baseline measurement to assess the scale targeting. If $15 \%$ of patients scored minimum or maximum points, the floor or ceiling effect was considered confirmed ${ }^{18}$.

To test cross-cultural validity, the differences in the motivation dimension scores between gender, educational and relationship status subgroups were assessed using independent samples t-test. Pearson correlation coefficients were calculated to assess the association between RM4-FM and sociodemographic characteristics of the participants. Furthermore, convergence with the PAAQ score was examined by calculating Pearson correlation coefficients for each dimension as well as for RAl against the PAAQ score. The coefficient values of $0.3,0.5,0.7$ and 0.9 were interpreted as low, moderate, strong and very strong correlation, respectively 22 . 
To assess the structural validity of RM4-FM, confirmatory factor analysis was conducted. The presumed factor structure consisting of four motivation dimensions was tested. The Chi-square test of model fit, the root mean square error of approximation (RMSEA), the comparative fit index (CFI) and the standardized root mean square residual (SRMR) were calculated. A non-significant chi-square test value, RMSEA less than 0.05 , CFI over 0.95 and SRMR less than 0.80 were interpreted as good fit of the tested model ${ }^{28}$. The unidimensionality of the RM4-FM dimensions were examined with exploratory factor analysis (EFA). In addition, a best-fit factor model was explored with EFA. Varimax rotation with the Kaiser normalization method was used to obtain the most distinguishable factor structure. A factor loading of 0.4 was used as a cutoff value representing that the item typifies the factor sufficiently 27.

Internal consistency of RM4-FM and its dimensions were assessed using Cronbach's alpha with bootstrapping method of 1000 repetitions to obtain the $95 \% \mathrm{Cls}^{6}$. Alpha values over 0.7 were considered as acceptable ${ }^{28}$.

Intraclass Correlation Coefficients (ICC) and 95\% confidence intervals were calculated to assess testretest-reliability. ICC over 0.7 was interpreted as sufficient stability, while ICC values under 0.7 represent unacceptable stability 28,33 .

The statistical analyses were conducted with R and SPSS 25.0 statistical software. Interpretation and reporting of the results of this study adhere to the COnsensus-based Standards for the selection of health status Measurement INstruments (COSMIN) guidelines ${ }^{28}$. The present study was conducted as part of a larger study investigating the effects of resistance training on functional capacity ${ }^{35}$, health ${ }^{13}$ and psychological well-being ${ }^{14}$. The study protocol was approved by The Ethical Committee of The University of Jyväskylä.

\section{Results}

\section{Translation}

RM4-FM translated well in to Finnish. The comparison of the two forward-translated versions of RM4-FM revealed minor linguistic differences that did not change the content of the questionnaire. In addition, the cross-checking of back-translated and the original version of the RM4-FM showed no substantial discrepancies. After minor adjustments, the translated version of RM4-FM was approved by the researchers (Appendix 1).

\section{Sample characteristics}

A total of 102 eligible participants completed the questionnaires in their entirety. Table 1 presents the sociodemographic and clinical characteristics of the participants. The mean body mass index (BMI) of the participants was 28 indicating mild overweight. While a proportion of participants undertook medication for blood pressure, cholesterol and/or blood glucose, none had serious injury or illness and 
could be considered healthy and cognitively proficient for this age group. Variation in the average duration of physical activity per week was high ranging from 0 to 200 minutes.

\section{Distribution of the RM4-FM}

Figure 1 presents the distributions of each RM4-FM dimensions. Distribution of External regulation score was skewed towards low scores while distributions of Identified regulation and Intrinsic motivation scores were skewed towards high scores. Introjected regulation score and RAI were normally distributed. The coverage of the distribution was good in Introjected regulation dimension while in other dimensions the skewness of the distribution impaired the coverage. A floor effect was confirmed in the External regulation dimension and a ceiling effect was confirmed in the Identified regulation dimension (Table 2). No other floor or ceiling effects were observed.

Table 2

Mean scores, Cronbach's alphas, and percentage of maximum and minimum points of RM4-FM dimensions.

\begin{tabular}{|lllll|}
\hline Dimension & Mean (SD) & Alpha (95\% Cl) & Minimum (\%) & Maximum (\%) \\
\hline External regulation & $2.3(1.3)$ & $0.82(0.72$ to 0.89$)$ & 21.6 & 2.0 \\
\hline Introjected regulation & $4.1(1.3)$ & $0.75(0.63$ to 0.83$)$ & 0 & 2.9 \\
\hline Identified regulation & $6.2(0.9)$ & $0.72(0.60$ to 0.80$)$ & 0 & 21.6 \\
\hline Intrinsic motivation & $5.7(1.1)$ & $0.84(0.76$ to 0.90$)$ & 0 & 11.8 \\
\hline
\end{tabular}

\section{Cross-cultural validity}

The examination of differences in motivation dimension scores and RAl between subgroups by gender revealed, that External regulation score was higher among male (2.6 vs. 2.1, $p=0.030$ ) and mean RAl of female participants was higher than in males (9.8 vs. $7.8, p=0.009)$. No other significant differences were observed between genders. The External regulation (1.9 vs. 2.6, $p=0.003$ ) and Introjected regulation (3.7 vs. $4.3, p=0.027$ ) scores of participants with higher education were lower compared to basic educated participants. Other dimension scores or RAI did not differ between educational subgroups. In addition, marital status was not associated to dimension scores or RAI.

The Pearson correlation coefficients of age, BMI and physical activity time with RM4-FM dimensions and RAl showed no notable correlations. Yet, the correlations of BMI with Introjected regulation score $(r=$ $-0.273, p=0.006)$ and Intrinsic motivation score $(r=-0.20, p=0.045)$, and of physical activity time with Intrinsic motivation score $(r=0.276, p=0.006)$, Identified regulation score $(r=0.21, p=0.034)$ and Introjected regulation score $(r=0.20, p=0.048)$ were statistically significant but still of negligible strength. PAAQ scores correlated positively to RAI $(r=0.422, p<0.001)$, Intrinsic motivation $(r=0.394, p<$ $0.001)$ and Identified regulation $(r=0.341, p<0.001)$ scores. Nevertheless, the strength of the correlations was low. 


\section{Structural validity}

The results of confirmatory factor analysis indicated poor fit of the tested four-dimension factor model of RM4-FM, as the Chi-square test of model fit $\left(X^{2}=256, d f=98, p<0.001\right)$, the RMSEA value $0.126(90 \% \mathrm{Cl}$ $=0.107$ to $0.145, p<0.001)$, the CFI value 0.811 and the SRMR 0.103 demonstrated inadequacy of the model. On the other hand, when unidimensionality of each RM4-FM dimension was tested separately with exploratory factor analysis (EFA), all motivation dimensions showed unidimensionality as only one factor with Eigenvalue over 1 was found for each dimension. Furthermore, all items in each dimension loaded strongly (loading value over 0.4 ) on these identified factors. Internal consistency of the RM4-FM was good as the Cronbach's alpha was 0.88 (95\% $\mathrm{Cl} 0.83$ to 0.91). In addition, the alphas of the RM4-FM dimensions showed acceptable internal consistency with values over 0.7 (range 0.72 to 0.84 ) (Table 2).

Examination of the best-fit factor model of RM4-FM with EFA revealed three factors with eigenvalues over 1 (Table 3). Factor 1 (Eigenvalue $=6.0$ ) consisted of all items of External regulation and one item of Introjected regulation dimensions, which all emphasize the influence of other people on motivation (items $2,6,7,11$ and 14). Factor 2 (Eigenvalue $=3.1$ ) consisted of two items of Intrinsic motivation and three items of Identified regulation dimensions (items 8, 9, 12, 15 and 16). The items of factor 2 concentrate on intention of well-being. Three items of Introjected regulation, two items of Intrinsic motivation and one item of Identified regulation dimensions formed factor 3 (Eigenvalue $=1.1$, items 1, 3, 4, 5, 10 and 13). Factor 3 incorporates the items on emotional aspect of motivation comprising the items on joy of physical activity and, on the other hand, guilt of inactivity. Cronbach's alphas of these factors were 0.86 , 0.85 and 0.81 for factors 1,2 and 3 , respectively. 
Table 3

Factor loadings of RM4-FM items in the best-fit factor model.

\begin{tabular}{|lllll|}
\hline Item number & Dimension & Factor 1 & Factor 2 & Factor 3 \\
\hline 1 & Introjected regulation & 0.23 & 0.27 & 0.54 \\
\hline 3 & External regulation & 0.80 & -0.03 & 0.17 \\
\hline 4 & Intrinsic motivation & -0.02 & 0.31 & 0.79 \\
\hline 5 & Introjected regulation & 0.50 & 0.16 & 0.60 \\
\hline 6 & Identified regulation & 0.06 & 0.25 & 0.71 \\
\hline 7 & Introjected regulation & 0.79 & 0.01 & 0.20 \\
\hline 9 & External regulation & 0.83 & 0.02 & 0.05 \\
\hline 10 & Intrinsic motivation & 0.23 & 0.61 & 0.47 \\
\hline 11 & Identified regulation & -0.10 & 0.67 & 0.40 \\
\hline 12 & Intrinsic motivation & -0.02 & 0.52 & 0.64 \\
\hline 13 & External regulation & 0.82 & -0.05 & 0.19 \\
\hline 14 & Identified regulation & 0.21 & 0.79 & 0.18 \\
\hline 15 & Introjected regulation & 0.36 & 0.17 & 0.52 \\
\hline 16 & External regulation & 0.73 & 0.32 & -0.18 \\
\hline
\end{tabular}

\section{Test-retest reliability}

Eighty-one participants (79\%) re-completed the RM4-FM. Figure 2 shows the baseline and repeated measure scores of RM4-FM dimensions. The ICC values of RM4-FM dimensions of Intrinsic motivation (ICC $=0.87,95 \% \mathrm{Cl}=0.79$ to 0.92$)$, External regulation $(\mathrm{ICC}=0.85,95 \% \mathrm{Cl}=0.76$ to 0.90$)$ and Identified regulation (ICC $=0.80,95 \% \mathrm{Cl}=0.68$ to 0.87 ) indicated good stability while ICC value of Introjected regulation (ICC $=0.78,95 \% \mathrm{Cl}=0.66$ to 0.86 ) showed moderate stability. Mean RAI remained stable in baseline and repeated measures (8.9 in baseline vs. 9.2 in repeated). The ICC of RAI (ICC $=0.86,95 \% \mathrm{Cl}=$ 0.78 to 0.91$)$ indicated good stability.

\section{Discussion}

The main finding of this study was that the Finnish version of RM4-FM provides valid and reliable scores in physical activity motivation assessment. Nevertheless, our findings showed limitations regarding the 
targeting of the dimension scores and a need for consideration of factor structure of RM4-FM (Table 4). 
Predefined hypotheses and conclusions for validation of the RM4-FM.

Feature

Coverage and

targeting

Floor and ceiling No floor effect (min score $\leq 15 \%$ )

effect

Intrinsic motivation

Identified regulation

Introjected regulation

External regulation

No ceiling effect (max score $\leq 15 \%)$

Intrinsic motivation

Identified regulation

Introjected regulation

External regulation

Validity

Cross-cultural validity

Convergence with the PAAQ
Non-significant associations between the RM4-FM score and sociodemographic characteristics

Significant and at least low correlation between the PAAQ and the RM4-FM scores

Conclusion

Confirmed

Confirmed

Confirmed

Rejected

Confirmed

Rejected

Confirmed

Confirmed

Confirmed

/Rejected

Confirmed

/Rejected

Structural validity

CFA

Chi-square test non-significant

Rejected

RMSEA $<0.05$

Rejected

$\mathrm{CFI}>0.95$

Rejected

CFA = Confirmatory Factor Analysis

$E F A=$ Exploratory Factor Analysis

RMSEA $=$ Root Mean Square Error of Approximation

$\mathrm{CFI}=$ Comparative Fit Index

SRMR = Standardized Root Mean Square Residual

ICC = Intraclass Correlation Coefficient 


\begin{tabular}{|c|c|c|}
\hline \multirow[t]{2}{*}{ Feature } & Hypothesis & Conclusion \\
\hline & SRMR $<0.80$ & Rejected \\
\hline \multirow[t]{5}{*}{ EFA } & Unidimensional structure of dimensions & \\
\hline & Intrinsic motivation & Confirmed \\
\hline & Identified regulation & Confirmed \\
\hline & Introjected regulation & Confirmed \\
\hline & External regulation & Confirmed \\
\hline \multirow{5}{*}{$\begin{array}{l}\text { Internal } \\
\text { consistency }\end{array}$} & Cronbach's alpha is $\geq 0.7$ & \\
\hline & Intrinsic motivation & Confirmed \\
\hline & Identified regulation & Confirmed \\
\hline & Introjected regulation & Confirmed \\
\hline & External regulation & Confirmed \\
\hline \multicolumn{3}{|l|}{ Reliability } \\
\hline \multirow{5}{*}{$\begin{array}{l}\text { Test-retest } \\
\text { reliability }\end{array}$} & $\mathrm{ICC} \geq 0.7$ & \\
\hline & Intrinsic motivation & Confirmed \\
\hline & Identified regulation & Confirmed \\
\hline & Introjected regulation & Confirmed \\
\hline & External regulation & Confirmed \\
\hline \multicolumn{3}{|c|}{ CFA $=$ Confirmatory Factor Analysis } \\
\hline \multicolumn{3}{|c|}{ EFA $=$ Exploratory Factor Analysis } \\
\hline \multicolumn{3}{|c|}{ RMSEA = Root Mean Square Error of Approximation } \\
\hline \multicolumn{3}{|c|}{$\mathrm{CFI}=$ Comparative Fit Index } \\
\hline \multicolumn{3}{|c|}{ SRMR = Standardized Root Mean Square Residual } \\
\hline ICC = Intracla & relation Coefficient & \\
\hline
\end{tabular}

There is a lack of validated instruments concerned with motivation of physical activity for the Finnish population. In this study, the translation and cross-cultural validation of the RM4-FM was performed according to internationally submitted guidelines ${ }^{37}$. There were only minor discrepancies between two 
forward translated versions, as well as between the original and backward translated versions, and these discrepancies did not affect the content of the instrument. Furthermore, the examination of the association of sociodemographic factors and RM4-FM scores indicated good cross-cultural validity of RM4-FM.

The scale targeting of RM4-FM dimensions was not optimal on the basis of the distributions and coverage of each dimension scores. The scores of the dimensions associated to internal motivation (Intrinsic motivation and Identified regulation) were skewed towards high scores and a ceiling effect was confirmed in Identified regulation dimension, whereas External regulation score was skewed towards low scores and was confirmed with a floor effect. The result suggests that applicability of RM4-FM is limited in a sample of highly internally motivated people. A study by Nurmi et al (2005) ${ }^{24}$ investigated the mediator effect of self-regulation in the association between intrinsic motivation and participation in physical activity in adolescents using a broadly similar questionnaire with minor differences (SRQ-E). The scores of Intrinsic motivation and Identified regulation dimensions in that study were focused around high scores with mean scores 3.84 (SD 0.97) and 3.85 (SD 0.96), respectively. On the other hand, the mean scores of External regulation and Introjected regulation were 1.78 (SD 0.85) and 2.78 (SD 1.09). The distributions of the scores in the study by Nurmi et al. ${ }^{24}$ and in the present study were very similar supporting the suggestion that scale targeting and ceiling effects might be a problem independent of age group.

The confirmatory factor analysis (CFA) showed that RM4-FM did not obey the presumed four-dimension structure. The results of CFA on the four-dimensional model indicated weak structural validity of RM4-FM. In addition, despite the sufficiency of Cronbach alphas of the RM4-FM and its dimensions, the values were relatively low, especially within the individual dimensions. Low Cronbach alphas of the dimensions may be due to a low number (4) of items in each dimension, but, on the other hand, it may reflect that items within the dimensions do not measure the same construct. The exploratory factor analysis (EFA) produced a best-fit model of three factors: influence of other people, intention of well-being and emotions of physical activity. The three-factor model showed at least comparable, if not better, performance in psychometric properties compared to the original model. However, despite the strengths of the threefactor model, it is limited due to losing the basis and division in motivation dimensions based on the Selfdetermination theory ${ }^{8}$. The three-factor model would provide specific information on motivation from a different perspective than the original model. Nevertheless, the EFA of single RM4-FM dimensions showed unidimensionality of each dimension, which in turn support the sufficiency of structural validity and applicability of the four-dimensional model of RM4-FM.

High ICC values indicated high test-retest reliability with one-month re-completion interval. Motivation for physical activity is a dynamic phenomenon that may vary depending on changing situations and mood of one's life. High ICC suggests that RM4-FM is not strongly affected by these varying circumstances, but it rather reflects more permanent aspects of motivation. 
There were some limitations in the present study. Firstly, criterion validity of RM4-FM was not measured, as there was not a sufficient instrument to compare the results. Secondly, the study sample was limited to older individuals (i.e. 65-75 years of age), which, in addition, may be influenced by a high rate of chronic diseases and medication use in the sample. The age and health status of the participants may have also affected habits and attitudes, as well as motivation for physical activity, particularly since the present sample were highly functionable for their age despite the fact that they were not physically activity. The advantages of this study were a large randomly selected sample from the Population Register, which represents well the average population as the regularly physically active individuals were excluded, as well as comprehensive translation and statistical methods, which adhere to the international guidelines on questionnaire validation studies. In addition, the questionnaires were completed electronically, and the questionnaire program required the participant to complete all the items, thus, preventing missing data.

In conclusion, the Finnish version of RM4-FM instrument provides valid and reliable scores in assessing motivation for physical activity in a sample of healthy, older Finnish adults. However, regarding the floor and ceiling effects of the dimensions, the scale seemed to be improperly targeted suggesting shortages in the scale when evaluating internally motivated subjects. A three-factor model of the RM4-FM provided more favorable psychometric properties than the original version though it loses the basis of SelfDetermination Theory. The three-factor model is a noteworthy alternative when assessing motivation for physical activity using the RM4-FM. This work may aid in future translations into other languages and uses of the questionnaire.

\section{Declarations}

\section{Ethics approval and consent to participate}

Ethical approval was granted by the Ethics committee of the University of Jyväskylä, Finland and all participants signed informed consent prior to the study initiation.

\section{Consent for publication}

Not applicable

\section{Availability of data and materials}

The datasets used and/or analyzed during the current study are available from the corresponding author on reasonable request.

\section{Competing interests}

The authors declare that they have no competing interests.

\section{Funding}


This work was funded by a personal grant to Dr. Simon Walker by the Ministry of Education and Culture, Finland (OKM/56/626/2014).

\section{Authors' contributions}

$\mathrm{AH}$ and SW designed the study and performed the linguistically translations. KG collected and analyzed the data. MU wrote the manuscript and performed further analyses. MU, JR, and AH interpreted the data. All authors read, edited and approved the manuscript.

\section{Acknowledgements}

Not applicable.

\section{References}

1. Bize R, Johnson JA, Plotnikoff RC. Physical activity level and health-related quality of life in the general adult population: a systematic review. Preventive medicine. 2007;45(6):401-415.

2. Blair SN. Physical inactivity: the biggest public health problem of the 21 st century. British journal of sports medicine. 2009;43(1):1-2.

3. Bloom DE, Cafiero E, Jané-Llopis E, et al. The global economic burden of noncommunicable diseases. Program on the Global Demography of Aging;2012.

4. Butryn ML, Arigo D, Raggio GA, Kaufman Al, Kerrigan SG, Forman EM. Measuring the ability to tolerate activity-related discomfort: initial validation of the physical activity acceptance questionnaire (PAAQ). Journal of Physical Activity and Health. 2015;12(5):717-726.

5. Chen L, Magliano DJ, Zimmet PZ. The worldwide epidemiology of type 2 diabetes mellitus-present and future perspectives. Nature reviews endocrinology. 2012;8(4):228.

6. Cronbach LJ. Coefficient alpha and the internal structure of tests. psychometrika. 1951;16(3):297334.

7. Deci EL RR. Exercise self-regulation questionnaires. Self-determination theory: An approach to human motivation and personality - the self-regulation questionnaires. 2004.

8. Deci EL, Ryan RM. Overview of self-determination theory: An organismic dialectical perspective. Handbook of self-determination research. 2002:3-33.

9. Deci EL, Ryan RM. Self-determination theory: A macrotheory of human motivation, development, and health. Canadian psychology/Psychologie canadienne. 2008;49(3):182.

10. Digelidis N, Papaioannou A, Laparidis K, Christodoulidis T. A one-year intervention in 7th grade physical education classes aiming to change motivational climate and attitudes towards exercise. Psychology of Sport and exercise. 2003;4(3):195-210. 
11. Haggar FA, Boushey RP. Colorectal cancer epidemiology: incidence, mortality, survival, and risk factors. Clinics in colon and rectal surgery. 2009;22(4):191.

12. Hernlund $\mathrm{E}$, Svedbom A, Ivergård $\mathrm{M}$, et al. Osteoporosis in the European Union: medical management, epidemiology and economic burden. Archives of osteoporosis. 2013;8(1-2):136.

13. Ihalainen JK, Inglis AJ, Mäkinen T, et al. Strength training improves metabolic health markers in older individual regardless of training frequency. Frontiers in Physiology. 2019;10:32.

14. Kekalainen T, Kokko K, Tammelin T, Sipila S, Walker S. Motivational characteristics and resistance training in older adults: A randomized controlled trial and 1-year follow-up. Scandinavian journal of medicine \& science in sports. 2018;28(11):2416-2426.

15. Kessler RC, Bromet EJ. The epidemiology of depression across cultures. Annual review of public health. 2013;34:119-138.

16. Kohl 3rd HW, Craig CL, Lambert EV, et al. The pandemic of physical inactivity: global action for public health. The lancet. 2012;380(9838):294-305.

17. Levesque CS, Williams GC, Elliot D, Pickering MA, Bodenhamer B, Finley PJ. Validating the theoretical structure of the Treatment Self-Regulation Questionnaire (TSRQ) across three different health behaviors. Health education research. 2006;22(5):691-702.

18. McHorney CA, Tarlov AR. Individual-patient monitoring in clinical practice: are available health status surveys adequate? Quality of Life Research. 1995;4(4):293-307.

19. Mokkink LB, Terwee CB, Knol DL, et al. The COSMIN checklist for evaluating the methodological quality of studies on measurement properties: a clarification of its content. BMC medical research methodology. 2010;10(1):22.

20. Mokkink LB, Terwee CB, Patrick DL, et al. The COSMIN checklist for assessing the methodological quality of studies on measurement properties of health status measurement instruments: an international Delphi study. Quality of life research. 2010;19(4):539-549.

21. Moustaka FC, Vlachopoulos SP, Vazou S, Kaperoni M, Markland DA. Initial validity evidence for the Behavioral Regulation in Exercise Questionnaire-2 among Greek exercise participants. European Journal of Psychological Assessment. 2010;26(4):269.

22. Mukaka MM. A guide to appropriate use of correlation coefficient in medical research. Malawi Medical Journal. 2012;24(3):69-71.

23. Murcia J, Gimeno EC, Camacho AM. Measuring self-determination motivation in a physical fitness setting: validation of the Behavioural Regulation in Exercise Questionnaire-2 (BREQ-2) in a Spanish sample. The Journal of Sport Medicine and Physical Fitness. 2007;47:366-378.

24. Nurmi J, Hagger MS, Haukkala A, Araújo-Soares V, Hankonen N. Relations Between Autonomous Motivation and Leisure-Time Physical Activity Participation: The Mediating Role of Self-Regulation Techniques. Journal of Sport and Exercise Psychology. 2016;38(2):128-137.

25. Pate RR, Pratt M, Blair SN, et al. Physical activity and public health: a recommendation from the Centers for Disease Control and Prevention and the American College of Sports Medicine. Jama. 1995;273(5):402-407. 
26. Penedo FJ, Dahn JR. Exercise and well-being: a review of mental and physical health benefits associated with physical activity. Current opinion in psychiatry. 2005;18(2):189-193.

27. Peterson RA. A meta-analysis of variance accounted for and factor loadings in exploratory factor analysis. Marketing Letters. 2000;11(3):261-275.

28. Prinsen CA, Mokkink LB, Bouter LM, et al. COSMIN guideline for systematic reviews of patientreported outcome measures. Quality of Life Research. 2018;27(5):1147-1157.

29. Ryan RM, Patrick H, Deci EL, Williams GC. Facilitating health behaviour change and its maintenance: Interventions based on self-determination theory. European Health Psychologist. 2008;10(1):2-5.

30. Sanchis-Gomar F, Perez-Quilis C, Leischik R, Lucia A. Epidemiology of coronary heart disease and acute coronary syndrome. Annals of translational medicine. 2016;4(13).

31. Silva MN, Vieira PN, Coutinho SR, et al. Using self-determination theory to promote physical activity and weight control: a randomized controlled trial in women. Journal of behavioral medicine. 2010;33(2):110-122.

32. Teixeira PJ, Silva MN, Mata J, Palmeira AL, Markland D. Motivation, self-determination, and long-term weight control. International Journal of Behavioral Nutrition and Physical Activity. 2012;9(1):22.

33. Terwee CB, Bot SD, de Boer MR, et al. Quality criteria were proposed for measurement properties of health status questionnaires. Journal of clinical epidemiology. 2007;60(1):34-42.

34. Townsend N, Wilson L, Bhatnagar P, Wickramasinghe K, Rayner M, Nichols M. Cardiovascular disease in Europe: epidemiological update 2016. European heart journal. 2016;37(42):3232-3245.

35. Turpela M, Häkkinen K, Haff GG, Walker S. Effects of different strength training frequencies on maximum strength, body composition and functional capacity in healthy older individuals. Experimental gerontology. 2017;98:13-21.

36. Wallhead TL, Ntoumanis N. Effects of a sport education intervention on students' motivational responses in physical education. Journal of teaching in physical education. 2004;23(1):4-18.

37. Wild D, Grove A, Martin M, et al. Principles of good practice for the translation and cultural adaptation process for patient-reported outcomes (PRO) measures: report of the ISPOR Task Force for Translation and Cultural Adaptation. Value in health. 2005;8(2):94-104.

38. Vos T, Barber RM, Bell B, et al. Global, regional, and national incidence, prevalence, and years lived with disability for 301 acute and chronic diseases and injuries in 188 countries, 1990-2013: a systematic analysis for the Global Burden of Disease Study 2013. The Lancet. 2015;386(9995):743800.

\section{Figures}



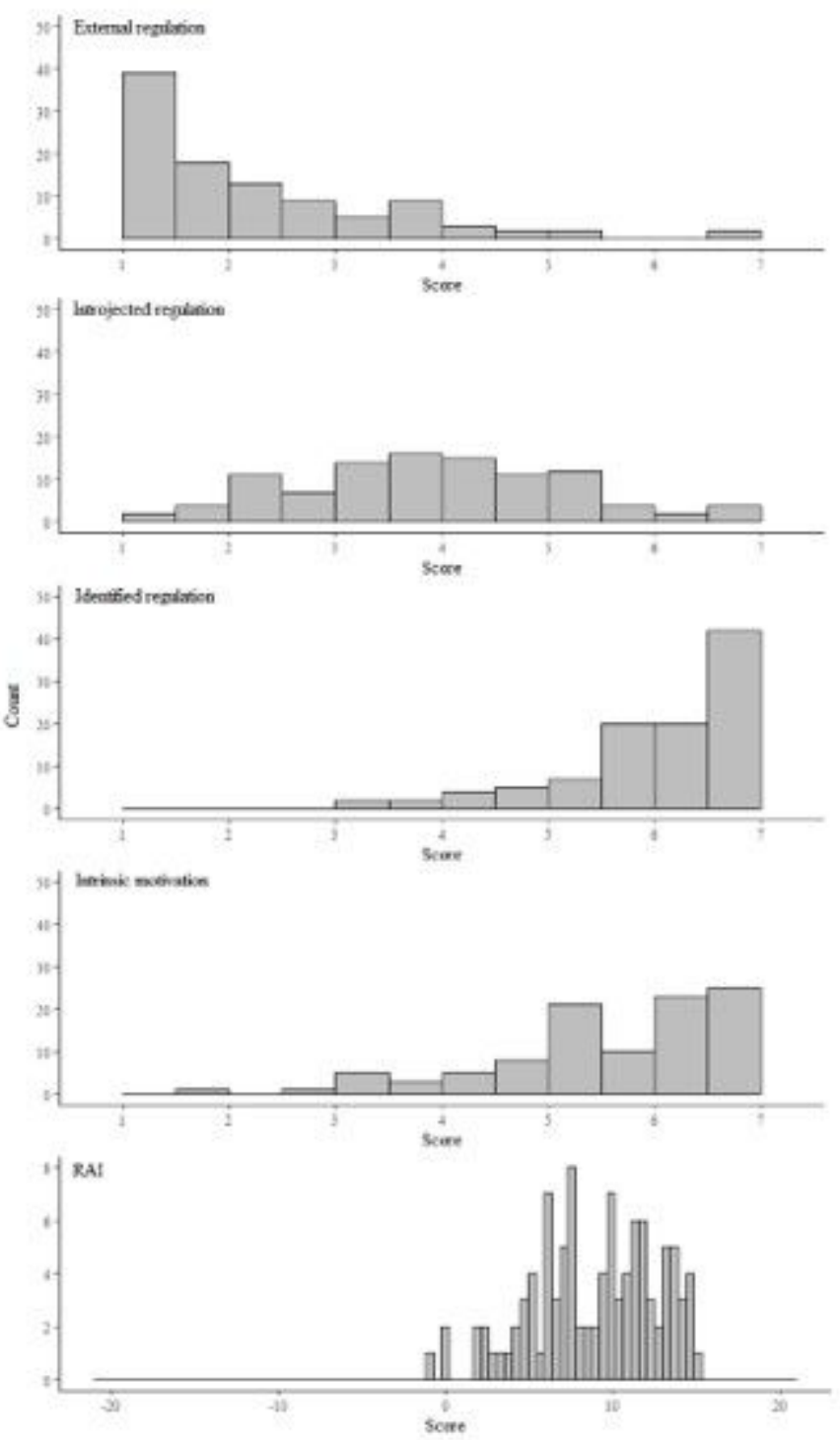

Figure 1

Distributions of individual RM4-FM dimension scores and RAI. 


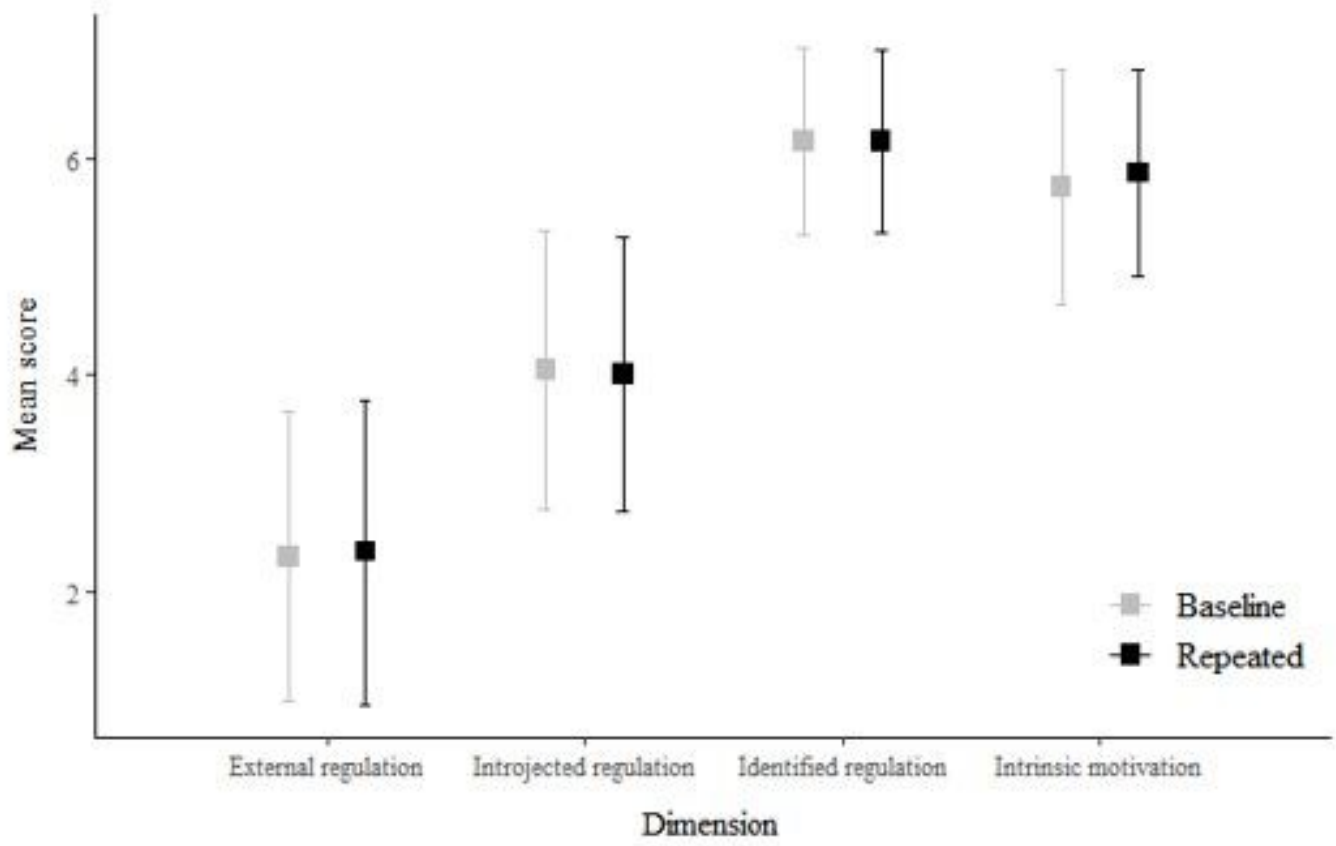

Figure 2

Comparison of baseline and repeated RM4-FM dimension scores.

\section{Supplementary Files}

This is a list of supplementary files associated with this preprint. Click to download.

- Appendix1.docx 